\title{
Nucleus-nucleus potential, energy dissipation and mass dispersion in fusion and transfer reactions
}

\author{
Kouhei Washiyama ${ }^{1, \mathrm{a}}$, Denis Lacroix ${ }^{1, \mathrm{~b}}$, and Sakir Ayik ${ }^{2, \mathrm{c}}$ \\ 1 GANIL, Bd Henri Becquerel, BP55027, 14076 Caen, France \\ 2 Physics Department, Tennessee Technological University, Cookeville, Tennessee 38505, USA
}

\begin{abstract}
The nucleus-nucleus potential and energy dissipation in fusion reactions are obtained from microscopic mean-field dynamics. The deduced potentials nicely reproduce the one extracted from experimental data. Energy dissipation shows a universal behaviour between different reactions. Also, the dispersion of mass distribution in transfer reaction is investigated in a stochastic mean-field dynamics. By including initial fluctuations in collective space, the description of the dispersion is much improved compared to that of mean field only. The result is consistent with the macroscopic phenomenological analysis of the experimental data.
\end{abstract}

\section{Introduction}

The interplay between nuclear structure and dynamical effects is crucial to properly describing nuclear reactions at energies close to the Coulomb barrier. Therefore, the theories describing such nuclear reactions need a unified description for both nuclear structure and dynamics. Moreover, recent developments on nuclear facilities introduce much interest on the properties of nuclei far from the stability. The time-dependent Hartree-Fock (TDHF) theory [1-4] based on the Skyrme energy density functional (EDF) provides a rather unique tool for describing nuclei over the whole nuclear chart. The TDHF theory solves the time evolution of single-particle wave functions according to

$$
i \hbar \frac{\partial}{\partial t} \rho=[h[\rho], \rho]
$$

where $h[\rho]$ denotes the self-consistent mean-field Hamiltonian from the Skyrme EDF, denoted by $\mathcal{E}[\rho]$, obtained from $h[\rho]=\delta \mathcal{E}[\rho] / \delta \rho$ with the one-body density $\rho$. This model automatically includes important dynamical effects such as vibrations of nuclei, neck formations, and nucleon transfer during reactions. Since recent computational developments now enable us to include all the terms of the Skyrme EDF used in static Hartree-Fock calculations in the three-dimensional coordinate space [5-8], the description of nuclear reactions using TDHF should be revisited.

In this contribution, as an illustration of applications of the TDHF theory to nuclear reactions, we discuss the properties of nucleus-nucleus potential and energy dissipation extracted from the TDHF model $[9,10]$. Moreover, we investigate fluctuations of one-body observables, especially, the dispersion of mass distributions in transfer reactions using a stochastic mean-field model $[11,12]$.

\footnotetext{
a e-mail: washiyama@ganil.fr

b e-mail: lacroix@ganil.fr

c e-mail: ayik@tntech.edu
}

\section{Nucleus-nucleus potential and energy dissipation from mean-field dynamics}

Nucleus-nucleus potential and energy dissipation are extracted as follows $[9,10]$ : (i) The TDHF equation for headon collision is solved to obtain the time evolution of the total density of colliding nuclei. (ii) From the total density, we compute at each time the relative distance $R$, associated momentum $P$, and reduced mass $\mu$ of colliding nuclei. (iii) We assume that the time evolutions of $R$ and $P$ obey a classical equation of motion including a friction term which depends linearly on the velocity $\dot{R}$ :

$$
\frac{d R}{d t}=\frac{P}{\mu}, \quad \frac{d P}{d t}=-\frac{d V}{d R}-\gamma(R) \dot{R},
$$

where $V(R)$ and $\gamma(R)$ denote the nucleus-nucleus potential and friction coefficient, respectively. The friction coefficient $\gamma(R)$ describes the effect of energy dissipation from the macroscopic collective degrees of freedom to the microscopic ones. For the TDHF calculations presented in this contribution, the three-dimensional TDHF code developed by P. Bonche and coworkers with the SLy4d Skyrme effective force [5] is used. The mesh sizes in space and in time are $0.8 \mathrm{fm}$ and $0.45 \mathrm{fm} / c$, respectively. For more details, see Refs. [9, 10].

Dynamical effect on potentials deduced from TDHF trajectories at center-of-mass energies close to the Coulomb barrier is seen in all reactions considered here. Figure 1 shows the difference between the barrier height deduced from TDHF trajectories $\left(V_{B}\right)$ and the barrier height extracted from experimental data $\left(V_{B}^{\exp }\right)[13,14]$ as a function of $V_{B}$. The solid line corresponds to the barrier height extracted using high-energy TDHF trajectories $\left(E_{\text {c.m. }} \gg V_{B}\right)$, whereas the dashed line is the result for low-energy TDHF trajectories $\left(E_{\text {c.m. }} \sim V_{B}\right)$. The former identifies with the barrier height of the frozen density apploximation [15]. Dynamical reduction of the barrier height from high-energy

This is an Open Access article distributed under the terms of the Creative Commons Attribution-Noncommercial License, which permits unrestricted use, distribution, and reproduction in any noncommercial medium, provided the original work is properly cited. 


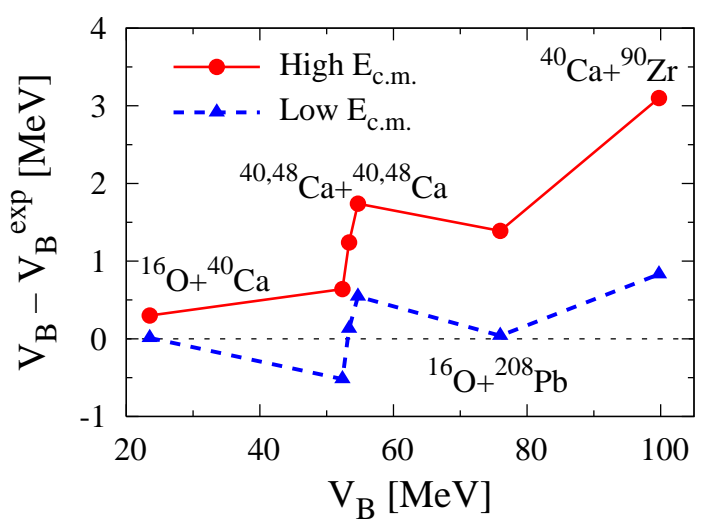

Fig. 1. Barrier height $V_{B}$ deduced from our method minus experimental barrier height $V_{B}^{\exp }$ as a function of extracted barrier height for the reactions indicated in the figure. The values $V_{B}$ are deduced from high energy (solid line) and from low energy (dashed line) TDHF trajectories, respectively.

TDHF to low-energy TDHF is clearly seen for all reactions. Moreover, because of this reduction, the value of the barrier height at low energy approaches the experimental data. This underlines the importance of dynamical effects close to the Coulomb barrier and shows the precision of our method.

Our method is also able to provide information on energy dissipation through the friction coefficient $\gamma$, which is shown in Fig. 2. In this figure, we present reduced friction coefficients $\beta(R) \equiv \gamma(R) / \mu$ as a function of $R$ scaled by the Coulomb barrier radius $R_{B}$ for different reactions. Figure 2 clearly shows that the order of magnitude of $\beta(R)$ and the radial dependence are almost independent on the size and asymmetry of the system. We also compare our results with that of a microscopic model based on small amplitude response by Adamian et al. [16] by the solid circles. The radial dependence and the magnitude of the friction coefficient are very similar to those extracted from our method.

From here, we conclude that the use of the macroscopic equation (2) is valid and mean-field dynamics gives good descriptions for the nucleus-nucleus potential and energy dissipation.

\section{Mean-field fluctuations}

It is well known that the mean-field model gives good descriptions for average evolution of one-body observables in low-energy nuclear reactions. However, it completely fails in the description of the dynamics of fluctuations of one-body observables. One of the shortcomings may be the failure of the mean-field description of the dispersion of mass distributions of final fragments in deep inelastic collisions. It has been recognized for a long time that TDHF calculations severely underestimate the dispersion of mass distributions of experiments $[17,18]$, although TDHF calculations well reproduce the mean value of fragment mass.

During the past decades, much effort has been devoted to overcoming this difficulty and to developing transport

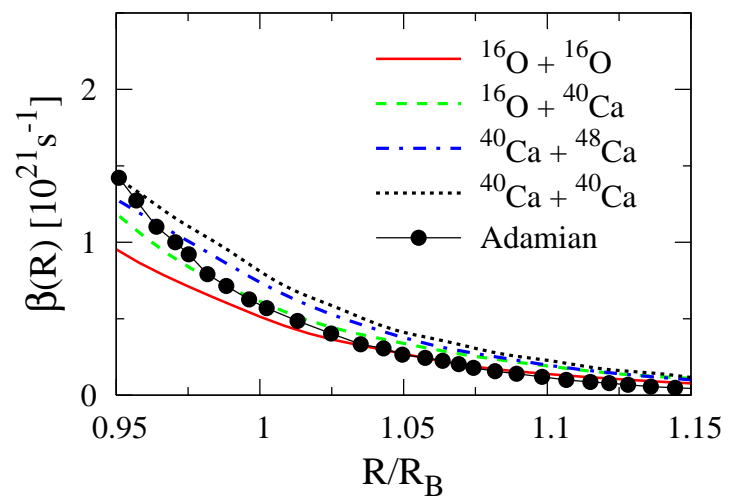

Fig. 2. Extracted reduced friction $\beta(R) \equiv \gamma(R) / \mu$ as a function of $R$ scaled by the Coulomb barrier radius $R_{B}$ for different reactions. A microscopic friction coefficient by Adamian et al. is shown by the solid circles for comparison.

theories that are able to describe not only mean values but also fluctuations (for a review, see Refs [19,20]). Among them, the variational principle by Balian and Vénéroni (BV) appears as one of the most promising methods [21-23]. However, even nowadays it remains difficult to apply [24]. More than 30 years after the first application of the TDHF theory, the absence of a practical solution to include fluctuations beyond mean field in a fully microscopic framework strongly restricts applications of mean-field-based theories.

In order to overcome this difficulty, recently we proposed a stochastic mean-field (SMF) approach, which is a stochastic extension of the mean-field model for low energy nuclear dynamics so as to include zero-point fluctuations of the initial state $[11,25]$. The initial density fluctuations are simulated by representing the initial state in terms of a suitable ensemble of initial single-particle density matrices, which is similar to the idea in Refs. [26,27]. In fact, this idea can be regarded as the beginning of constructing time-dependent version of configuration mixing calculations. In this manner, the description with single Slater determinant is replaced by a superposition of multi Slater determinants. A member of the ensemble, indicated by event label $\lambda$, can be expressed as

$$
\rho^{\lambda}\left(\mathbf{r}, \mathbf{r}^{\prime}, t\right)=\sum_{i j \sigma \tau} \Phi_{i \sigma \tau}^{*}(\mathbf{r}, t ; \lambda) \rho_{i j}^{\lambda}(\sigma \tau) \Phi_{j \sigma \tau}\left(\mathbf{r}^{\prime}, t ; \lambda\right)
$$

where summations $i$ and $j$ run over a complete set of singleparticle wave functions $\Phi_{i \sigma \tau}(\mathbf{r}, t ; \lambda)$, and $\sigma$ and $\tau$ denote spin and isospin quantum numbers. According to the description of the SMF approach [25], the elements of density matrices $\rho_{i j}^{\lambda}(\sigma \tau)$ are assumed to be time-independent random Gaussian numbers with mean value $\overline{\rho_{i j}^{\lambda}(\sigma \tau)}=\delta_{i j} n_{i}^{\sigma \tau}$ and with the variance of the fluctuating part $\delta \rho_{i j}^{\lambda}(\sigma \tau)$ specified by

$$
\begin{aligned}
& \overline{\delta \rho_{i j}^{\lambda}(\sigma \tau) \delta \rho_{j^{\prime} i^{\prime}}^{\lambda}\left(\sigma^{\prime} \tau^{\prime}\right)} \\
= & \frac{1}{2} \delta_{j j^{\prime}} \delta_{i i^{\prime}} \delta_{\tau \tau^{\prime}} \delta_{\sigma \sigma^{\prime}}\left[n_{i}^{\sigma \tau}\left(1-n_{j}^{\sigma \tau}\right)+n_{j}^{\sigma \tau}\left(1-n_{i}^{\sigma \tau}\right)\right] .
\end{aligned}
$$


Here, $n_{i}^{\sigma \tau}$ denotes the average single-particle occupation factor. At zero temperature occupation factors are 0 and 1 , and at finite temperature they are determined by the Fermi-Dirac distribution. The great advantage of the SMF approach is that each Slater determinant evolves independently from each other following the time evolution of its single-particle wave functions in its self-consistent meanfield Hamiltonian, denoted by $h\left(\rho^{\lambda}\right)$, according to

$$
i \hbar \frac{\partial}{\partial t} \Phi_{i \sigma \tau}(\mathbf{r}, t ; \lambda)=h\left(\rho^{\lambda}\right) \Phi_{i \sigma \tau}(\mathbf{r}, t ; \lambda) .
$$

In the following applications, we focus on the head-on ${ }^{40} \mathrm{Ca}+{ }^{40} \mathrm{Ca}$ collision around the Coulomb barrier energy.

\subsection{Fusion reactions}

First, we apply the SMF approach to fusion reactions [11]. To discuss the fluctuation of collective variables, we map the SMF time evolution to a one-dimensional macroscopic Langevin equation, which is similar to Eq. (2) except an additional Gaussian random force $\xi_{P}^{\lambda}(t)$ :

$$
\frac{d}{d t} P^{\lambda}=-\frac{d}{d R^{\lambda}} U\left(R^{\lambda}\right)-\gamma\left(R^{\lambda}\right) \dot{R}^{\lambda}+\xi_{P}^{\lambda}(t),
$$

Ignoring non-Markovian effects, the random force $\xi_{P}^{\lambda}(t)$ with zero mean value reduces to white noise specified by a correlation function,

$$
\overline{\xi_{P}^{\lambda}(t) \xi_{P}^{\lambda}\left(t^{\prime}\right)}=2 \delta\left(t-t^{\prime}\right) D_{P P}(R) .
$$

Here $D_{P P}(R)$ denotes the momentum diffusion coefficient. We note that the expression of the diffusion coefficient has the same form as that obtained from the phenomenological nucleon exchange model [28]. As an example, the diffusion coefficient for the head-on ${ }^{40} \mathrm{Ca}+{ }^{40} \mathrm{Ca}$ collision at $E_{\text {c.m. }}=100 \mathrm{MeV}$ is shown in Fig. 3 .

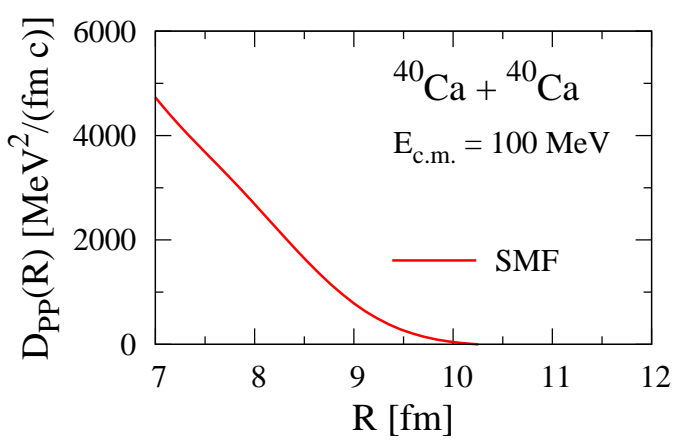

Fig. 3. Diffusion coefficient as a function of the relative distance for the head-on ${ }^{40} \mathrm{Ca}+{ }^{40} \mathrm{Ca}$ collision at $E_{\text {c.m. }}=100 \mathrm{MeV}$.

\subsection{Mass dispersion in transfer reactions}

Another application has been made to the dispersion of the fragment mass distribution to improve the mean-field description [12]. To do so, we investigate transfer reactions near the Coulomb barrier, where nucleon exchange will occur during reaction, and estimate the dispersion of the fragment mass distribution.

In the SMF approach, time evolution of the mass number of the projectile-like fragment $A_{P}^{\lambda}$ is also described by a Langevin equation [29],

$$
\frac{d}{d t} A_{P}^{\lambda}=v\left(A_{P}^{\lambda}, t\right)+\xi_{A}^{\lambda}(t)
$$

where $v\left(A_{P}^{\lambda}, t\right)$ denotes the drift term for nucleon transfer. The Gaussian white noise random force $\xi_{A}^{\lambda}(t)$ is determined with zero mean value and with a correlation function,

$$
\overline{\xi_{A}^{\lambda}(t) \xi_{A}^{\lambda}\left(t^{\prime}\right)}=2 \delta\left(t-t^{\prime}\right) D_{A A},
$$

where $D_{A A}$ is the diffusion coefficient associated with nucleon exchange. The variance $\sigma_{A A}^{2}$ of fragment mass distribution is determined by small fluctuations of the mass number $\delta A_{P}^{\lambda}$ through $\sigma_{A A}^{2}(t)=\overline{\delta A_{P}^{\lambda} \delta A_{P}^{\lambda}}$. According to the Langevin equation, neglecting contributions from the drift term, the variance is related to the diffusion coefficient according to $[29,30]$

$$
\sigma_{A A}^{2}(t) \simeq 2 \int_{0}^{t} D_{A A}(s) d s .
$$

In the phenomenological nucleon exchange model, the relation $\sigma_{A A}^{2}(t)=N_{\text {exc }}(t)$ was obtained, where $N_{\text {exc }}(t)$ denotes the accumulated total number of exchanged nucleons until time $t$, and was extensively used to analyze the experimental data of mass dispersion [31]. In the following, to check whether the SMF approach satisfies the above relation, we estimate the both quantities by the SMF approach.

In Fig. 4, the variances of the fragment mass distributions deduced from the SMF approach for the head-on

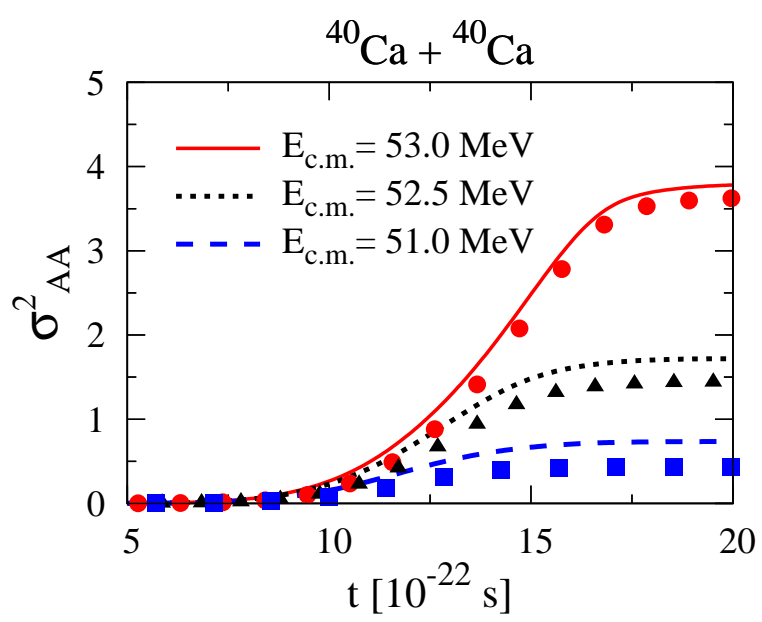

Fig. 4. Time evolution of $\sigma_{A A}^{2}$ obtained from the SMF approach for ${ }^{40} \mathrm{Ca}+{ }^{40} \mathrm{Ca}$ reaction at different center-of-mass energies. Number of exchanged nucleon is superimposed by the solid circles, solid triangles, and solid squares from high to low energies. 
${ }^{40} \mathrm{Ca}+{ }^{40} \mathrm{Ca}$ reaction at three center-of-mass energies are shown by lines. The number of exchanged nucleons is inserted in Fig. 4 by the solid circles, solid triangles, and solid squares from high to low energies. The mass variance estimated from the SMF approach is consistent with this relation. We also estimate the variance of fragment mass distribution using the standard TDHF approach. The asymptotic values of $\sigma_{A A}^{2}$ for the ${ }^{40} \mathrm{Ca}+{ }^{40} \mathrm{Ca}$ reaction are $0.004,0.008$, and 0.008 from low to high energies, while the number of exchanged nucleons are $0.432,1.441$, and 3.634. The TDHF results are much smaller than the number of exchanged nucleons and are also much smaller than the results obtained from the SMF approach that are 0.730 , $1.718,3.790$. The failure of the TDHF theory on the description of variances of the fragment mass distribution has been recognized for a long time as a major limitation of the mean-field theory. It appears that the SMF approach cures this shortcoming. As seen from Fig. 4, not only the asymptotic value of $\sigma_{A A}^{2}$ but also the entire time evolution is very close to the evolution of $N_{\text {exc }}(t)$.

\section{Conclusion}

Mean-field dynamics and mean-field fluctuations using microscopic time-dependent models are discussed in the context of low energy nuclear reactions. We have shown that the TDHF theory gives precise values of nucleus-nucleus potential and a universal behavior of energy dissipation. We have also shown that the SMF approach correctly describes the mass dispersion of final fragments in transfer reaction at energies near the Coulomb barrier. This gives a practical solution to properly describe mean-field fluctuations on top of mean field.

This work is supported in part by US DOE Grant DE-FG0589ER40530.

\section{References}

1. P. Bonche, S. E. Koonin, and J. W. Negele, Phys. Rev. C 13, (1976) 1226.

2. S. E. Koonin, Prog. Part. Nucl. Phys. 4, (1980) 283.

3. J. W. Negele, Rev. Mod. Phys. 54, (1982) 913.

4. C. Simenel, B. Avez, and D. Lacroix, Lecture notes of the "International Joliot-Curie School", Maubuisson, September 17-22, 2007, arXiv:0806.2714.

5. K.-H. Kim, T. Otsuka, and P. Bonche, J. Phys. G 23, (1997) 1267.

6. T. Nakatsukasa and K. Yabana, Phys. Rev. C 71, (2005) 024301.

7. A. S. Umar and V. E. Oberacker, Phys. Rev. C 73, (2006) 054607.

8. J. A. Maruhn, P.-G. Reinhard, P. D. Stevenson, and M. R. Strayer, Phys. Rev. C 74, (2006) 027601.

9. K. Washiyama and D. Lacroix, Phys. Rev. C 78, (2008) 024610.

10. K. Washiyama, D. Lacroix, and S. Ayik, Phys. Rev. C 79, (2009) 024609.
11. S. Ayik, K. Washiyama, and D. Lacroix, Phys. Rev. C 79, (2009) 054606.

12. K. Washiyama, S. Ayik, and D. Lacroix, Phys. Rev. C 80, (2009) 031602(R).

13. L. C. Vaz, J. M. Alexander, and G. R. Satchler, Phys. Rep. 69, (1981) 373.

14. J. O. Newton et al., Phys. Lett. B586, (2004) 219; Phys. Rev. C 70, (2004) 024605.

15. V. Y. Denisov and W. Nörenberg, Eur. Phys. J. A 15, (2002) 375 .

16. G. G. Adamian, R. V. Jolos, A. K. Nasirov, and A. I. Muminov, Phys. Rev. C 56, (1997) 373.

17. K. T. R. Davies, V. Maruhn-Rezwani, S. E. Koonin, and J. W. Negele, Phys. Rev. Lett. 41, (1978) 632.

18. C. H. Dasso, T. Døssing, and H. C. Pauli, Z. Phys. A 289, (1979) 395.

19. Y. Abe, S. Ayik, P.-G. Reinhard, and E. Suraud, Phys. Rep. 275, (1996) 49.

20. D. Lacroix, S. Ayik, and Ph. Chomaz, Prog. Part. Nucl. Phys. 52, (2004) 497.

21. R. Balian and M. Vénéroni, Phys. Lett. B136, (1984) 301.

22. R. Balian, P. Bonche, H. Flocard, and M. Vénéroni, Nucl. Phys. A428, (1984) 79c.

23. J. B. Marston and S. E. Koonin, Phys. Rev. Lett. 54, (1985) 1139.

24. J. M. A. Broomfield and P. D. Stevenson, J. Phys. G 35, (2008) 095102.

25. S. Ayik, Phys. Lett. B658, (2008) 174.

26. H. Esbensen, A. Winther, R. A. Broglia, and C. H. Dasso, Phys. Rev. Lett. 41, (1978) 296.

27. C. H. Dasso and R. Donangelo, Phys. Lett. B276, (1992) 1.

28. H. Feldmeier, Rep. Prog. Phys. 50, (1987) 915.

29. J. Randrup and W. J. Swiatecki, Ann. Phys. (N.Y.) 125, (1980) 193; Nucl. Phys. A429, (1984) 105.

30. J. Randrup, Nucl. Phys. A383, (1982) 468.

31. H. Freiesleben and J. V. Kratz, Phys. Rep. 106, (1984) 1. 